\title{
Escala de Identificação das Altas Habilidades/Superdotação: Evidências de Validade de Critério
}

\author{
Giftedness Identification Scale: Evidence of Criterion Validity
}

\author{
Priscila Zaia ${ }^{1}$ e Tatiana de Cássia Nakano ${ }^{2}$
}

\begin{abstract}
Resumo
O objetivo desse estudo foi investigar evidências de validade baseadas nas relações com outras variáveis validade de critério concorrente para a Escala de Identificação de Características associadas às Altas Habilidades/Superdotação (EICAH/S). A amostra foi composta por dois grupos, sendo que 405 estudantes de uma cidade do interior do Estado de São Paulo compuseram o grupo controle e 80 crianças provenientes dos Estados de Santa Catarina e Distrito Federal que frequentavam o NAAH/S e as salas de recursos de atendimento às altas habilidades/superdotação $(\mathrm{AH} / \mathrm{S})$ constituíram o grupo critério. Os participantes responderam à EICAH/S, cujos resultados apontaram para médias significativamente superiores a favor do grupo critério nos dois fatores (cognitivo e socioemocional) e na pontuação total da escala, demostrando a eficácia da escala em discriminar os indivíduos com e sem indicadores de AH/S.
\end{abstract}

Palavras-chave: superdotados, escalas, avaliação psicológica, validade do teste

\begin{abstract}
The aim the current research was to investigate the evidence of validity based on relationship with other variables - concurrent criterion validity of the Scale to Identify Giftedness Characteristics (SIGC). The sample was composed by two groups. 405 students from a city of São Paulo State composed the control group and 80 children from Santa Catarina and Distrito Federal States, which attended the NAAH/S and a resource classroom to gifted children constituted the criterion group. The participants answered the SIGC and the results indicated higher averages obtained by the criterion group. The results showed that the scale discriminates the groups with or without giftedness indicators.
\end{abstract}

Keywords: gifted students, scales (testing), psychological assessment, test validity

\footnotetext{
${ }^{1}$ Pós-doutoranda em Psicologia na Universidade São Francisco (USF). Ex-aluna do curso de pós-graduação stricto sensu em Psicologia da Pontifícia Universidade Católica de Campinas. Brasil. Av. John Boyd Dunlop, s/n. - Jardim Ipaussurama. CEP 13060-904, Campinas, SP, Brasil. Tel.: +55 19 98189-4046. E-mail: priscilazaia@ gmail.com

${ }^{2}$ Docente do curso de pós-graduação stricto sensu em Psicologia da Pontifícia Universidade Católica de Campinas. Brasil. 


\section{Introdução}

$\mathrm{O}$ interesse em compreender as habilidades superiores apresentadas pelos indivíduos com altas habilidades/superdotação (AH/S) tem crescido ao longo dos anos. Ideias equivocadas acerca dos construtos envolvidos na conceituação, as quais consideravam apenas uma pequena parcela de potencialidades (notadamente associadas aos altos níveis de inteligência) têm perdido foco (Sternberg, 2018; Stoeger, Balestrini, \& Ziegler, 2018) para dar espaço às concepções multidimensionais, mais abrangentes e atuais (Renzulli \& Reis, 2018). Consequentemente, o reconhecimento de diferentes áreas de desenvolvimento humano em que as habilidades superiores podem se apresentar tem predominado na atualidade (Subotnik, Olszewski-Kubilius, \& Worrell, 2018).

Segundo as leis nacionais, os estudantes com potencial elevado em uma ou mais áreas do conhecimento humano, sejam elas de criatividade, liderança, psicomotora, artística, intelectual, acadêmica e que apresentem grande envolvimento com tarefas de seu interesse, são considerados superdotados e incluídos como público-alvo da Educação Especial (Brasil, 2012; Chacon, Pedro, Koga, \& Soares, 2017). Dada a diversidade de áreas em que a alta habilidade/superdotação pode se manifestar, bem como a possibilidade de que ela ocorra em somente uma área ou mais de uma, uma grande diversidade de traços apresentados por esses indivíduos tem sido apontada. Notadamente, a literatura científica tem destacado a importância de se considerar a interação entre fatores cognitivos e aspectos socioemocionais, assim como, as condições ambientais e culturais, as quais podem favorecer ou impedir o desenvolvimento dos potenciais elevados (Neihart \& Yeo, 2018; Stoeger et al., 2018).

Sendo assim, pesquisadores têm ressaltado que as características envolvidas no fenômeno podem ser tanto do tipo cognitivas quanto não cognitivas. As do primeiro tipo podem envolver habilidades relacionadas à resolução de problemas, raciocínio abstrato, habilidades acadêmicas, além de associar-se aos aspectos cognitivos da inteligência, sendo facilmente mensuradas por testes de desempenho. Por outro lado, as do tipo não cognitivo, podem incluir aspectos emocionais, afetivos e sociais, podendo ser relacionadas tanto às habilidades socioemocionais quanto às provenientes da inteligência emocional, tais como percepção, expressão, compreensão, regulação de estados emocionais e sentimentos sobre si mesmo e sobre os outros, não sendo um tipo excludente do outro (Gagné, 2018; Jones, Greenberg, \& Crowley, 2016; Rodrigo-Ruiz, Cejudo, \& Pérez-González, 2019).

Nesse sentido, reconhecer a diversidade de características que podem se apresentar acima da média em um indivíduo é, sem dúvida, um dos primeiros passos a ser dado na percepção da presença de altas habilidades/superdotação (Wellisch \& Brown, 2012). À vista das ampliações e atualizações conceituais, faz-se importante que os processos de identificação de indivíduos com potencial elevado acompanhem as evoluções dos modelos teóricos. Para isso, a literatura científica tem argumentado sobre a necessidade da utilização de diferentes métodos capazes de mensurar a diversidade de perfis existentes dentre essa população (Renzulli \& Reis, 2018), incluindo instrumentos que avaliem tanto as aptidões gerais e cognitivas quanto habilidades específicas e traços não cognitivos.

Cada método contribui com uma parcela do processo, sendo que, quando utilizados em conjunto, podem permitir a identificação de diferentes tipos de potencialidades. No entanto, na prática, a identificação dos indivíduos que apresentam potencialidades elevadas tem enfrentado uma série de dificuldades dada a variedade de comportamentos apresentados, aos diversos níveis de superdotação, aos diferentes modelos teóricos que abarcam a conceituação do tema, à influência dos fatores socioeconômicos e culturais, aos questionamentos acerca da forma como a identificação deve ocorrer, aos métodos que vêm sendo utilizados e às dúvidas sobre quais comportamentos podem ser selecionados (Mun, 2016; Virgolim, 2013; Wellisch \& Brown, 2012). Quando uma ou mais dessas variáveis se faz presente, os desafios se iniciam, visto que, para ser reconhecido como indivíduo superdotado e ser incluído nos dados do censo escolar, cujos dados são tomados como base para a formulação de políticas públicas no Brasil, o processo de identificação precisa ocorrer de maneira correta e 
íntegra, a fim de evitar a invisibilidade desses indivíduos na sociedade (Ourofino \& Fleith, 2011; Pérez \& Freitas, 2014).

Com o objetivo de minimizar essa problemática, os especialistas na área têm indicado que o processo de identificação dessa população deve ser feito com base no uso de diferentes tipos de instrumentos. Isto é, tanto aqueles capazes de avaliar as aptidões gerais quanto as específicas do fenômeno, tal como testes de habilidades e desempenho, recomendações externas, checklist de indicadores, dentre outros métodos e procedimentos, cada um com sua própria carga de importância (Kim \& Berebitsky, 2016; Ziegler, Stoeger, \& Vialle, 2012). Assim, ressalta-se que todo esse procedimento deve englobar tanto as diversas áreas de conhecimento quanto aquelas ligadas às emoções e atitudes, visto que de forma isolada, os testes de inteligência não compreendem esferas do comportamento, expressões artísticas, musicais, entre outras. Por sua vez, as listas de indicadores, atualmente utilizadas através do preenchimento dos pais, professores, colegas ou pela própria criança também não são suficientes (Renzulli \& Reis, 2018; Vieira, 2014). Tal situação evidencia a necessidade de instrumentos desenvolvidos especificamente para uso nessa população, dentro de uma bateria mais ampla que empregue diferentes metodologias e ferramentas. Somente assim a identificação poderá contemplar a heterogeneidade de perfis dos indivíduos superdotados, bem como, seus diferentes níveis de habilidades (Hertzog et al., 2018).

Nesse sentido, Kieffer, Reese e Vacha-Haase (2010) argumentam que a preocupação no campo das altas habilidades/superdotação deve se voltar ao desenvolvimento de medidas específicas aos indivíduos superdotados, as quais sejam capazes de capturar as diferenças individuais apresentadas por eles. Tal preocupação se mostra relevante se considerarmos que, quando esses indivíduos são avaliados com instrumentos desenvolvidos para a população geral, facilmente podem atingir o efeito teto (nível máximo que o teste consegue medir). No entanto, dentre aqueles indivíduos que se encontram no topo das habilidades, ainda existem diferentes níveis, que apenas os instrumentos específicos para essa população poderão ser capazes de inferir. Entretanto, a realidade brasileira ainda se mostra marcada, até o momento, pela inexistência de instrumentos aprovados pelo Conselho Federal de Psicologia, no Brasil, para uso específico na população de indivíduos com características superiores à média. Consequentemente, os processos de identificação e à instrumentalização dos profissionais se mostra marcado por uma importante lacuna.

Diante desse quadro, o processo de construção da Escala de Identificação de Características associadas às Altas Habilidades/Superdotação (EICAH/S) foi iniciado. Essa escala foi construída para ser utilizada como instrumento de rastreio de comportamentos superdotados em crianças, com idades entre 09 a 12 anos. Seu diferencial se baseia no formato de autorrelato, o qual oferece a possibilidade de o indivíduo apontar e avaliar as suas próprias características.

Junte-se a esse ponto $o$ fato de que instrumentos de triagem de comportamentos superdotados têm sido construídos e utilizados para complementar as avaliações realizadas pelos testes de desempenho no contexto internacional (Pfeiffer \& Petscher, 2008; Pfeiffer, Petscher, \& Jarosewich, 2007; Pfeiffer, Petscher, \& Kumtepe, 2008). Seu uso se dá como um primeiro passo no procedimento de identificação, caracterizando-se por itens com afirmações auto descritivas, que possibilitam avaliar a intensidade de determinado comportamento através das opções de respostas do tipo Likert, podendo-se destacar, dentre suas vantagens, a fácil administração (Kazem, Alzubaidi, Hemdan, \& Renzulli, 2014) e a oportunidade dos indivíduos demonstrarem sua percepção acerca de suas próprias características (Nunes et al., 2008).

No que diz respeito aos estudos psicométricos já realizados com a EICAH/S, destacam-se as evidências de validade de conteúdo, evidências de validade por meio da estrutura interna, bem como a investigação da precisão pelo coeficiente Alfa de Cronbach. O primeiro investigou o conteúdo dos itens através da participação de cinco juízes especialistas em avaliação psicológica e construção de instrumentos, estudantes de pósgraduação, os quais avaliaram se os itens se adequavam às características correspondentes (Bassinello, 2014). Os resultados mostraram-se favoráveis às evidências de validade de conteúdo para a referida escala, com a maioria dos itens 
acima de $80 \%$ de concordância e coeficientes Kappa acima de .75 para todos os juízes.

$\mathrm{O}$ segundo estudo utilizou a análise fatorial exploratória para verificar como os itens se agrupariam. A solução fatorial indicou a existência de dois fatores, que explicariam $40.4 \%$ da variância total. Dessa forma, a estrutura da escala foi dividida em Fator 1 - Características Socioemocionais e Fator 2 - Características Cognitivas. Por sua vez, o estudo de investigação da fidedignidade indicou coeficientes Alfa de Cronbach de .93 para o Fator 1 e .91 para o Fator 2, sendo ambos considerados excelentes (Zaia, Nakano, \& Peixoto, 2018).

Nesse sentido, o estudo aqui apresentado teve, como objetivo, apresentar evidências complementares de validade, notadamente, de critério. Esta fonte de evidências de validade usualmente é realizada a fim de investigar a eficácia com que o instrumento consegue predizer o desempenho de um indivíduo (Souza, Alexandre, \& Guirardello, 2017), em relação a diagnóstico ou diferenciação de grupos específicos (Pasquali, 2009).

Sendo assim, seguindo as recomendações da literatura científica, o presente estudo investigou as evidências de validade de critério concorrente da EICAH/S, a partir da sua aplicação em dois grupos de estudantes, sendo o grupo critério constituído por crianças com diagnóstico de altas habilidades/superdotação e o grupo controle por crianças que não possuíam nenhum tipo de condição diferenciada.

\section{Método}

\section{Participantes}

Colaboraram com este estudo 485 crianças, selecionadas por conveniência, as quais foram divididas em grupo critério e grupo controle. A amostra do grupo critério se constituiu por 80 participantes, sendo 58 do sexo masculino $(72,5 \%)$, estudantes do $3^{\circ}, 4^{\circ}$ e $5^{\circ}$ ano do ensino fundamental I $(43,9 \%)$ e do $6^{\circ}, 7^{\circ}, 8^{\circ}$ e $9^{\circ}$ ano do ensino fundamental II $(56,1 \%)$. A faixa etária da amostra foi de 09 a 12 anos $(M=10.66 ; D P=1.12)$. Para compor esse grupo foram selecionadas apenas crianças que, em algum momento, haviam passado por avaliação psicológica prévia, confirmando o quadro de altas habilidades/superdotação. Tal grupo frequentava os Núcleos de Atividades de Altas Habilidades/Superdotação do Estado de Santa Catarina e as salas de recurso para atendimento de AH/S no Distrito Federal.

Por sua vez, o grupo controle foi composto por 405 crianças, sendo 208 do sexo feminino (51.5\%), estudantes do $3^{\circ}, 4^{\circ}$ e $5^{\circ}$ ano do ensino fundamental I (53.5\%) e do $6^{\circ}$ e $7^{\circ}$ ano do ensino fundamental II (46.5\%), com idades entre 09 e 12 anos $(M=10.91 ; D P=.88)$. As crianças estavam matriculadas em sala de aula regular e não apresentavam diagnóstico de AH/S.

\section{Instrumento}

$\mathrm{O}$ instrumento utilizado nesse estudo foi a Escala de Identificação de Características associadas às Altas Habilidades/Superdotação (EICAH/S), desenvolvida por Zaia e Nakano (2014). A escala se caracteriza pelo formato de autorrelato, sendo composta por 38 afirmações a serem respondidas em uma escala tipo Likert de 4 pontos, agrupados em dois fatores: Fator 1 Características Socioemocionais e Fator 2 Características Cognitivas.

As opções de respostas são "Não tem nada a ver comigo", "Não tem muito a ver comigo", "Tem pouco a ver comigo" e "Tem muito a ver comigo". Os estudantes devem ler cada uma das frases e escolher uma das alternativas, indicando o quanto concordam com o conteúdo presente na afirmação.

$\mathrm{O}$ conjunto de itens da escala, constitui-se em comportamentos representativos de 22 características comumente apresentadas por indivíduos identificados como superdotados, selecionadas após revisão de literatura científica nacional e internacional (ver Bassinello, 2014). As características englobados na escala são: pensamento moral, capacidade de questionar, bom autoconceito/autoconfiança, perfeccionismo, interesses amplos, liderança, persistência, senso de humor, observação aguçada, curiosidade, capacidade de oferecer ajuda, criatividade/imaginação, capacidade de envolverse com a tarefa/motivação, fluência verbal, capacidade para leitura, intensidade emocional/sensibilidade com o outro, pensamento abstrato/complexo/lógico/perspicaz, capacidade para aprendizagem precoce e rápida, raciocínio 
superior/resolução de problemas, sucesso em atividades extracurriculares, capacidade de extrapolar conhecimentos para situações novas/ ir além do que é ensinado, boa memória.

\section{Procedimentos}

Três escolas públicas de uma cidade do interior do Estado de São Paulo foram contatadas e autorizaram a pesquisa em seus estabelecimentos. Com isso, seus alunos compuseram o grupo controle. É importante ressaltar que por motivo de ausência de identificação, poderiam ser encontradas crianças, nesses locais, que apresentassem indicadores da presença de $\mathrm{AH} / \mathrm{S}$, porém, o requisito para esse grupo era não ter avaliação psicológica previamente realizada com essa finalidade. Por sua vez, para localização das crianças do grupo critério, a pesquisadora realizou reuniões com os responsáveis por núcleos de atendimento específico para crianças identificadas com $\mathrm{AH} / \mathrm{S}$, assim como com professores que trabalham em salas de recursos específicos para essa população as quais funcionam dentro de escolas municipais de diferentes cidades do país, para suplementação de conteúdo, no contra turno escolar.

Nesse sentido, o grupo critério foi composto por estudantes que recebiam atendimento especializado em dois diferentes estados do país (Santa Catarina e Distrito Federal). Uma parte da amostra frequentava o núcleo e a outra era atendida em salas de recursos específicas para indivíduos com AH/S. É importante destacar que todos os indivíduos superdotados foram avaliados por psicólogos, com aplicação de testes de inteligência e criatividade, bem como, por professores, tendo sido realizado um processo multidisciplinar para o fechamento do diagnóstico de altas habilidades/superdotação. É importante ressaltar o rigor metodológico de seleção e inclusão dos estudantes nesses programas de atendimento.

As aplicações da EICAH/S ocorreram coletivamente em ambos os grupos, com duração aproximada de 40 minutos para os alunos do ensino fundamental I, do grupo controle, pelo fato da pesquisadora precisar ler alguns itens para que todos compreendessem as informações. Por outro lado, tanto as crianças do ensino fundamental II, do grupo controle, quanto as crianças do grupo critério, responderam a escala com tempo aproximado de 20 minutos.

\section{Análise de dados}

Os escores obtidos pelos participantes dos dois grupos (controle e critério), nos dois fatores e na pontuação total da escala foram estimados. Com o auxílio do programa Statistical Package for the Social Sciences (SPSS), versão 22, os primeiros testes realizados referiram-se à normalidade e homogeneidade da amostra, a fim de decidir se a comparação das médias dos grupos seria realizada por testes paramétricos ou nãoparamétricos (Field, 2009; Miot, 2017). Os resultados do teste de Kolmogorov-Smirnov apontaram dados significativos para o grupo critério e não significativos para o grupo controle. Por sua vez, o teste de Shapiro-Wilk mostrou dados não significativos para ambos os grupos. Portanto, o teste de Mann-Whitney, uma análise não-paramétrica de comparação de médias, foi adotado, considerando que a amostra deste estudo não atendeu os princípios da normalidade.

À vista disso, a estatística descritiva e tamanho do efeito através do d de Cohen foram realizadas, para consolidar a hipótese de que a escala em construção é capaz de diferenciar crianças identificadas das não identificadas com altas habilidades/superdotação. Para interpretação do tamanho do efeito, as recomendações de EspíritoSanto e Daniel (2015) foram seguidas: valores menores que .19 são insignificantes, valores entre .20 e .49 são pequenos; os médios oscilam entre .50 e .79 e, por sua vez, os valores entre .80 e 1.29 são grandes e os acima de 1.30, muito grandes.

\section{Aspectos éticos}

Para a condução desse estudo, os trâmites burocráticos relacionados ao Comitê de Ética foram cumpridos, sendo as reuniões com as instituições de ensino agendadas posteriormente à aprovação do projeto (CAAE 67963717.4.0000.5481). Os responsáveis pelas escolas, núcleo de atendimento e salas de recurso assinaram a Carta de Autorização para pesquisa em seus estabelecimentos. Por sua vez, os pais ou responsáveis pelas crianças participantes do estudo assinaram o Termo de Consentimento Livre e Esclarecido e as crianças com idades acima de 10 anos assinaram o Termo de Assentimento. 
Quadro 1. Médias e Desvios-Padrão para o Grupo Critério e Controle

\begin{tabular}{llccc}
\hline \multirow{2}{*}{ Grupos } & & \multicolumn{3}{c}{ Medidas da EICAH/S } \\
\cline { 2 - 4 } & & $\begin{array}{c}\text { Características } \\
\text { Socioemocionais (Fator 1) }\end{array}$ & $\begin{array}{c}\text { Características } \\
\text { Cognitivas (Fator 2) }\end{array}$ & $\begin{array}{c}\text { Total de itens } \\
\text { EICAH/S }\end{array}$ \\
\hline Grupo Critério & $\mathrm{M}$ & 78.00 & 44.29 & 122.29 \\
$(\mathrm{n}=80)$ & $\mathrm{DP}$ & 9.72 & 6.75 & 15.19 \\
\hline Grupo Controle & $\mathrm{M}$ & 73.72 & 38.38 & 112.09 \\
$(\mathrm{n}=405)$ & $\mathrm{DP}$ & 10.51 & 7.70 & 16.14 \\
\hline Total & $\mathrm{M}$ & 74.42 & 39.36 & 113.77 \\
$(\mathrm{n}=485)$ & $\mathrm{DP}$ & 10.49 & 7.86 & 16.41 \\
\hline
\end{tabular}

Quadro 2. Comparação de Médias Pelo Teste de Mann-Whitney Para os Fatores da EICAH/S e Tamanho de Efeito (d de Cohen)

\begin{tabular}{cccc}
\hline & Características Socioemocionais & Características Cognitivas & Total de itens \\
& (Fator 1) & (Fator 2) & EICAH/S \\
\hline $\mathrm{U}$ & 12233.000 & 8966.000 & 9897.500 \\
$\mathrm{Z}$ & -3.492 & -6.321 & -5.503 \\
$p$ & $.0001^{* *}$ & $.0001^{* *}$ & $.0001^{* *}$ \\
$\mathrm{~d}$ & $\mathrm{~d}=.42 ; p \leq .001$ & $\mathrm{~d}=.82 ; p \leq .001$ & $.65 ; p \leq .001$ \\
\hline
\end{tabular}

Legenda: U=Mann-Whitney; $* * p \leq .001 ; Z=$ desvio padrão; $\mathrm{d}=\mathrm{d}$ de Cohen.

\section{Resultados}

A fim de compreender as diferenças entre os escores obtidos pelo grupo critério e grupo controle, a estatística descritiva para cada um dos fatores, bem como na soma total da EICAH/S foi realizada. Os resultados são apresentados na Quadro 1. Os resultados descritos no Quadro 1 apontaram para melhor desempenho dos participantes do grupo critério nas duas medidas da escala, bem como em seu total, em comparação ao desempenho obtido pelos participantes do grupo controle. Com o objetivo de verificar se a diferença de médias encontrada entre os grupos foi significativa, o teste de Mann-Whitney foi empregado. Os resultados apontaram para valores significativos para as todas medidas da escala, conforme pode ser visualizado na Quadro 2.

Desse modo foi possível verificar que o grupo critério apresentou médias significativamente mais altas tanto nas características socioemocionais (Fator 1), cujo tamanho do efeito pode ser classificado como pequeno ( $\mathrm{d}=.42$; $p \leq .001$ ), nas características cognitivas (Fator 2) com tamanho de efeito considerado grande $(\mathrm{d}=.82 ; p \leq .001)$ e na pontuação total da escala (tamanho médio de efeito, $\mathrm{d}=.65 ; p \leq .001$ ).

\section{Discussão}

O objetivo do presente estudo, no qual era esperada a capacidade de discriminação de características associadas às altas habilidades/ superdotação da EICAH/S foi atingido. Isso pode ser afirmado, a partir dos resultados descritos, os quais apontaram para desempenho superior e significativo do grupo estabelecido como critério nas duas medidas analisadas (características socioemocionais e características cognitivas), bem como no total de itens, corroborando a eficácia da escala em diferenciar indivíduos identificados e não identificados. Os resultados desse tipo de estudo permitem compreender os limites e alcances do instrumento, informando, ao profissional, quais decisões ele pode ou não tomar com base nos resultados (Ambiel \& Carvalho, 2017). Nesse caso aqui específico, identificar a presença de indicadores de altas habilidades/superdotação.

A relevância do estudo aqui apresentado se justifica perante o fato de que esse tipo de evidência de validade tem sido considerada um dos requisitos mais importantes na construção de medidas psicológicas (American Psychological Association, American Educational Research Association, \& National Council on Measurement in Education, 2014; Pacico \& Hutz, 2015; Pasquali, 2009), de maneira que seus resultados, somados aos anteriormente obtidos, apontam para evidências positivas de validade para a escala em construção. Essa fonte de evidências de validade busca conhecer a relação entre os componentes do próprio teste e outros indicadores externos. No estudo aqui apresentado, buscou-se verificar a relação entre a pontuação do participante nas 
medidas cognitivas e socioemocionais, bem como da escala total com o desempenho do sujeito na vida real (diagnóstico de $\mathrm{AH} / \mathrm{S}$ ). Os resultados mostraram que a $\mathrm{EICAH/S}$ enquanto medida de indicadores socioemocionais e cognitivos se mostrou eficaz em diferenciar os grupos, identificando, corretamente, um melhor desempenho obtido pelos participantes do grupo critério.

A importância desses resultados se justifica perante a constatação de que um dos desafios existentes no processo de desenvolvimento dos talentos, é a dificuldade de implementar métodos de identificação que considerem a heterogeneidade de indivíduos superdotados, bem como, seus diferentes níveis de habilidades (Hertzog, Mun, DuRuz, \& Holiday, 2018). Também Kieffer et al. (2010) chamam a atenção para o fato de que, no campo das altas habilidades/superdotação, uma preocupação deve se voltar ao desenvolvimento de medidas capazes de capturar as diferenças individuais apresentadas por aqueles indivíduos que se encontram no topo dos níveis de habilidades visto que, conforme anteriormente salientado, é bastante comum o fato de que tais indivíduos atinjam o valor máximo das tabelas normativas dos instrumentos que foram desenvolvidos para a população geral. Consequentemente, podem não discriminar corretamente os diferentes níveis de habilidades de indivíduos superdotados. Essa informação confirma a importância do desenvolvimento de medidas específicas para avaliar as habilidades superiores.

Considerando-se que os resultados do estudo aqui relatado, mais especificamente as diferenças significativas entre os grupos avaliados com a $\mathrm{EICAH/S}$, pode-se afirmar que a escala consegue diferenciar, de forma adequada, tanto aqueles indivíduos que apresentam habilidade cognitiva acima da média quanto os que apresentam habilidades socioemocionais elevadas. Essa proposta vai ao encontro de uma série de recomendações encontradas na literatura científica na área, de que a avaliação dessa população deve envolver não só habilidades cognitivas (Almeida, Araújo, Sainz-Gómez, \& Prieto, 2016; Matthews \& Peters, 2018), como também, forças e traços associados às habilidades sociais e emocionais de cada indivíduo (Gagné, 2018; Hertzog et al., 2018; Renzulli \& Reis, 2018).
O formato escolhido (escala de autorrelato) mostrou-se adequado ao propósito para o qual foi construída, bem como a ideia de constituir-se em uma ferramenta de triagem, dentro de uma bateria mais ampla de avaliação das AH/S (Carman, 2013; Morisano \& Shore, 2010; Prieto, Sainz, \& Fernández, 2012; Veiga, 2014), complementar as avaliações realizadas, principalmente, por meio dos resultados obtidos em testes psicométricos (Pfeiffer \& Petscher, 2008; Pfeiffer et al., 2007; Pfeiffer et al., 2008).

Outra constatação importante que adiciona valor ao instrumental se ampara no fato de que, no Brasil, até o momento, nenhum teste voltado à identificação das $\mathrm{AH} / \mathrm{S}$ se encontra disponível para uso profissional. Ainda que o processo de construção de uma bateria composta por testes de desempenho em medidas de inteligência e criatividade, com esse objetivo específico possa ser localizado na literatura científica (Nakano et al., 2015; Nakano, Primi, Ribeiro, \& Almeida, 2016; Ribeiro, Nakano, \& Primi, 2014), bem como a construção de uma escala de avaliação externa para ser respondida por professores (Nakano, Campos, \& Santos, 2016; Nakano, Gozzoli, Alves, Zaia, \& Campos, 2016), nenhum desses instrumentais aborda a questão sob a perspectiva do próprio sujeito. Esse é o diferencial da escala de autorrelato aqui analisada.

Nesse sentido, torna-se relevante ponderar também os aspectos positivos associados às autoavaliações que os indivíduos podem fazer de si mesmos. Esse tipo de instrumento é caracterizado por sua praticidade de aplicação e mensuração de resultados, além da motivação envolvida nos processos de resposta, já que os respondentes podem achar interessante falar sobre si mesmos (Paulhus \& Vazire, 2009). Portanto, o objetivo desse tipo de metodologia na identificação dos comportamentos e habilidades relacionadas ao construto, relaciona-se não só à sua fácil administração, mas também à possibilidade de ser o primeiro passo das investigações, ou seja, tornar-se um auxílio à triagem inicial (Kazem et al., 2014).

\section{Considerações finais}

O tema das altas habilidades/superdotação tem sido amplamente estudado e aprofundado, em termos teóricos, culminando em concepções que 
reconhecem tanto a existência de domínios gerais quanto específicos de habilidades, associados à diferentes áreas do conhecimento humano. Entretanto, lacunas na prática cotidiana de indivíduos pertencentes a essa população, no Brasil, ainda se fazem presentes, notadamente aquelas relacionadas à identificação dessa população. A falta de instrumentos disponíveis para uso profissional que contribuam para um processo de identificação abrangente é um dos pontos principais a ser explorado.

Partindo dessa percepção, o estudo aqui apresentado visou acrescentar evidências de validade a outras já obtidas anteriormente para um instrumento em processo de desenvolvimento: a escala de indicadores de características associadas às altas habilidades/superdotação. Durante $o$ processo de construção de um instrumento, devese ter em mente a ideia de que a busca por evidências de validade é um processo, que deve ser marcado pelo acúmulo de evidências provenientes de diferentes fontes de validade.

Os resultados encontrados permitiram afirmar evidências de validade baseadas nas relações com outras variáveis, mais especificamente, a validade de critério, de modo a confirmar a eficácia desse instrumento em discriminar indivíduos com indicadores de $\mathrm{AH} / \mathrm{S}$ daqueles que não possuem tais características, em relação a aspectos socioemocionais e cognitivos, bem como possibilidade de auxiliar na diminuição da lacuna acima referida. Dessa forma, os resultados aqui apresentados podem ser somados aos estudos previamente realizados com a EICAH/S, aproximando-se da possibilidade de disponibilização profissional do instrumental e seu uso como ferramenta auxiliar nos processos de triagem de comportamentos associados ao tema, dentro de uma perspectiva de uma bateria ampla de avaliação.

Faz-se importante, entretanto, ressaltar as limitações e desafios encontrados para que o presente estudo fosse conduzido. Especificamente no que diz respeito aos participantes, considerando que o instrumento em estudo possui o objetivo de discriminar crianças identificadas com altas habilidades/superdotação daquelas que não apresentam essas características, foi necessário compor o grupo critério com indivíduos previamente avaliados como superdotados, dentro de procedimentos rigorosos de identificação. Essa condição dificultou o acesso a esse grupo, visto que a ausência de procedimentos de identificação dessa população nas escolas de ensino regular se faz presente na maior parte das instituições educacionais, somada ao desconhecimento do fenômeno e de suas características por parte de professores e diretores, bem como a presença de concepções errôneas sobre o indivíduo com essa condição. Consequentemente, houve a necessidade de buscá-las em locais de atendimento específico, tais como Núcleos de Atividades de Altas Habilidades e Superdotação (NAAH/S) e salas de recursos, os quais ainda não são oferecidos em todas as cidades do Brasil. Tal quadro, aliado ao pequeno número de crianças e adolescentes identificados e em processo de atendimento fez com que o processo de coleta de dados durasse, aproximadamente, um ano para ser completado.

No que diz respeito à escala, os desafios associados à estrutura de autorrelato adotada para a EICAH/S, também devem ser mencionados. À vista disso, pode-se observar certas tendências, apontada pela literatura científica, as quais caracterizam-se como um dos maiores vieses enfrentados na utilização desse formato de instrumento. Os mais comuns são conhecidos como: (1) desejabilidade social, a qual envolve algumas situações que podem forçar o indivíduo a descrever-se de forma exageradamente positiva, a fim de se sentir aceito; (2) aquiescência, que faz com que os indivíduos concordem com o item sem se importar com seu conteúdo; e (3) respostas extremas, a qual consiste na escolha de respostas extremas na escala de avaliação, seja a menor opção ou a maior. Essas situações apontam para a necessidade de que outros estudos sejam conduzidos visando identificar a influência desses fatores no resultado dos participantes.

Por fim, ressalta-se a importância de realizar estudos voltados à investigação da precisão e de normatização, bem como a investigação da influência de variáveis como sexo, idade e escolaridade no desempenho dos sujeitos. Tais estudos permitirão que todos os requisitos mínimos exigidos pelo Conselho Federal de Psicologia sejam atendidos e, consequentemente, o instrumento possa ser disponibilizado, futuramente, para uso profissional. Almeja-se que 
os resultados aqui apresentados possam contribuir para auxiliar no conhecimento dos indivíduos com AH/S de maneira multidimensional, considerando sua diversidade de características e possibilidades de atendimento educacional especializado.

\section{Referências}

Almeida, L. S., Araújo, A. M., Sainz-Goméz, M., \& Prieto, M. D. (2016). Challenges in the identification of giftedness: Issues related to psychological assessment. Anales de Psicología, 32(3), 621-627. doi:10.6018/analesps.32.3.259311.

Ambiel, R. A. M., \& Carvalho, L. F. (2017). Validade e precisão de instrumentos de avaliação psicológica. In M. R. C. Lins \& J. C. Borsa (Orgs.), Avaliação psicológica: Aspectos teóricos e práticos (pp. 115-125). Petrópolis, RJ: Vozes.

American Psychological Association, American Educational Research Association \& National Council on Measurement in Education (2014). Standards for educational and psychological testing. Washington: American Psychological Association.

Bassinello, P. Z. (2014). Construção de Escala de Autorrelato para Identificação de características associadas à Superdotação. Dissertação de Mestrado, Pontifícia Universidade Católica de Campinas, Campinas, SP, Brasil.

Brasil (2012) Secretaria de Educação Especial MEC. Políticas Públicas para Alta Habilidade/ Superdotação. Recuperado de: http://www.senado.gov.br/web/comissoes/CE/ AP/AP20080626_superdotados_Cl\%C3\%A1u diaGriboski.pdf.

Carman, C. A. (2013). Comparing apples and oranges: Fifteen years of definitions of giftedness in research. Journal of Advanced Academics, 24(1), 52-70. doi:10.1177/1932202X12472602.

Chacon, M. C. M., Pedro, K. M., Koga, F. O., \& Soares, A. A. S. (2017). Variáveis pessoais de professores e a inclusão de alunos com altas habilidades/superdotação. Revista Educação Especial, $\quad 30(59), \quad$ 775-786. doi: $10.5902 / 1984686$ X28433.
Espírito-Santo, H., \& Daniel, F. (2015). Calcular e apresentar tamanhos do efeito em trabalhos científicos (1): As limitações do $p<.05$ na análise de diferenças de médias de dois grupos. Revista Portuguesa de Investigação Comportamental e Social 2015, 1(1), 3-16. doi:10.31211/rpics.2018.4.2.97.

Field, A. (2009). Descobrindo a estatística usando o SPSS. Porto Alegre, RS: Artmed.

Gagné, F. (2018). Academic talent development: Theory and best practices. In S. I. Pfeiffer (Ed.), APA Handbook of Giftedness and Talent (pp. 163-183). Washington, DC: American Psychological Association. doi:10.1037/0000038-011.

Hertzog, N. B., Mun, R. U., DuRuz, B., \& Holiday, A. A. (2018). Identification of strengths and talents in young children. In. S. I. Pfeiffer (Ed.), APA Handbook of Giftedness and Talent (pp. 301-316). Washington, DC: American Psychological Association. doi:10.1037/0000038-011.

Jones, D. E., Greenberg, M., \& Crowley, M. (2016). How children's social behaviors relate to success in adulthood. The WERA Educational Journal, 8 (2), 27-33. Retirado de: https://www.weraweb.org/assets/docs/WEJ-May-2016-final.pdf

Kazem, A. M., Alzubaidi, A. S., Hemdan, A. H., \& Renzulli, J. (2014). The factor structure of the Scales for Rating the Behavioural Characteristics of Superior Students (SRBCSS): Results on an omani sample. International Journal for Talent Development and Creativity, 2 (1), 127-136. Retirado de: https://www.researchgate.net/profile/Laura_So $\mathrm{kal} /$ publication/297196498_Desjardin_N_Ellio tt_M_Sokal_S_Christle_S_Kornelsen_A_Nikk el_K_Lone_K_Sokal_L_2014_Transformative _learning_through_international_service_work _International_Journal_of_Talent_Developmen t_and_Creativity_/links/58f90c970f7e9ba3ba4 c610b/Desjardin-N-Elliott-M-Sokal-S-ChristleS-Kornelsen-A-Nikkel-K-Lone-K-Sokal-L2014-Transformative-learning-throughinternational-service-work-InternationalJournal-of-Talent-Development.pdf\#page $=127$

Kieffer, MK. M., Reese, R. J., \& Vacha-Haase, T. (2010). Reliability generalization methods in the context of giftedness research. In B. 
Thompson \& R. F. Subotnik (Orgs.), Methodologies for Conducting Research on Giftedness (pp. 89-111). Washington, DC: American Psychological Association. doi:10.1037/12079-005.

Kim, S., \& Berebitsky, D. (2016). An application of multivariate generalizability in selection of mathematically gifted students. Eurasia Journal of Mathematics, Science \& Technology Education, 12(9), 2323-2334. doi:10.12973/eurásia.2016.1269a.

Matthews, M. S., \& Peters, S. J. (2018). Methods to increase the identification rate of students from traditionally underrepresented populations for gifted services. In S. I. Pfeiffer (Ed.), APA Handbook of Giftedness and Talent (pp. 318-331. Washington, DC: American Psychological Association. doi:10.1037/0000038-021.

Miot, H. A. (2017). Avaliação da normalidade dos dados em estudos clínicos e experimentais. Jornal Vascular Brasileiro, 16(2), 88-91. doi:10.1590/1677-5449.041117.

Morisano, D., \& Shore, B. M. (2010). Can Personal Goal Setting Tap the Potential of the Gifted Underachiever? Roeper Review, 32, 249-258. doi:10.1080/02783193.2010.508156.

Mun, R. U. (2016). Identifying and assessing highly capable students. The WERA Educational Journal, 8(2), 14-17. Retirado de: https://www.wera-web.org/assets/docs/WEJMay-2016-final.pdf

Nakano, T. C., Campos, C. R., \& Santos, M. V. (2016). Escala de avaliação de altas habilidades/superdotação - versão professor: Validade de conteúdo. Estudos Interdisciplinares em Psicologia, 7(1), 103123. doi:10.5433/2236-6407.2016v7n1p103.

Nakano, T. C., Gozzolli, M. Z., Alves, R. J. R., Zaia, P., \& Campos, C. R. (2016). Investigación de la eficacia de una Escala de Evaluación de Altas Habilidades - versión profesor. Revista de Estudios y Experiencias en Educación, 15(29), 83-94. doi:10.21703/rexe.20162983945.

Nakano, T. C., Primi, R., Abreu, I. C. C., Gozzoli, M. Z., Caporossi, D. C., Miliani, A. F. M., \& Martins, A. A. (2015). Bateria para avaliação das altas habilidades/superdotação: Análise dos itens via Teoria de Resposta ao Item.
Estudos de Psicologia (Campinas), 32, 729741. doi:10.1590/0103-166x2015000400016.

Nakano, T. C., Primi, R., Ribeiro, W. J., \& Almeida, L. S. (2016). Evaluación multidimensional de la superdotación: Criterios de validez de la Batería de Inteligencia y Creatividad para predecirlos talentos artísticos y académicos. Anales de Psicología, 32, 628-637.

doi:10.6018/analesps.32.3.259391.

Neihart, M., \& Yeo, L. S. (2018). Psychological issues unique to the gifted student. In S. I. Pfeiffer (Ed.), APA Handbook of Giftedness and Talent (pp. 497-510). Washington, DC: American Psychological Association. doi:10.1037/0000038-032

Nunes, C. H. S. S., Primi, R., Nunes, M. F. O., Muniz, M., Cunha, T. F., \& Couto, G. (2008). Teoria de Resposta ao Item para otimização de escalas tipo likert - um exemplo de aplicação. Revista Ibero-americana de Diagnóstico y Evaluación - e Avaliação Psicológica, 1(25), 51-79. Retirado de: http://aidep.org/sites/default/files/201707/R253.pdf.

Ourofino, V. T. A. T., \& Fleith, D. S. (2011). A condição underachieverment em superdotação: Definição e características. Psicologia: Teoria e Prática, 13(3), 206-222. Retirado de: http://pepsic.bvsalud.org/pdf/ptp/v13n3/v13n3a 16.pdf.

Pacico, J. C., \& Hutz, C. S. (2015). Validade. In C. S. Hutz, D. R. Bandeira, \& C. M. Trentini (Orgs.), Psicometria (pp. 71-84). Porto Alegre, RS: Artmed.

Pasquali, L. (2009). Psicometria. Revista da Escola de Enfermagem da USP, 43 (esp), 992999. doi:10.1590/S0080-62342009000500002.

Paulhus, D. L., \& Vazire, S. (2009). The SelfReport Method. In R. W. Robins, R. C. Fraley, \& R. F. Krueger (Eds.), Handbook of research methods in personality psychology (pp.224-239). New York: Guilford.

Pérez, S. G. P. B., \& Freitas, S. N. (2014). Políticas públicas para as Altas Habilidades / Superdotação: Incluir ainda é preciso. Revista Educação Especial, 27(50), 627-640. doi:10.5902/1984686X14274.

Pfeiffer, S. I., \& Petscher, Y. (2008). Identifying young gifted children using the Gifted Rating 
Scales-Preschool/Kindergarten Form. Gifted Child Quarterly, 52(1), 19-29. doi:10.1177/0016986207311055.

Pfeiffer, S. I., Petscher, Y., \& Jarosewich, T. (2007). The Gifted Rating ScalesPreschool/Kindergarten Form: An analysis of the standardization sample based on age, gender and race. Roeper Review, 29(3), 206211. doi:10.1080/02783190801955418.

Pfeiffer, S. I., Petscher, Y., \& Kumtepe, A. (2008). The Gifted Rating Scales-School Form: A validation study based on age, gender and race. Roeper Review, 30, 140-146. doi:10.1080/02783190801955418.

Prieto L., Sainz, M., \& Fernández, M. C. (2012). Estudio de la superdotación em España. Revista Amazônica, X (3), 48-78. Retirado de: https://dialnet.unirioja.es/servlet/articulo?codig $\mathrm{o}=4047332$

Renzulli, J. S., \& Reis, S. M. (2018). The Threering conception of giftedness: A developmental approach for promoting creative productivity in young people. In S. I. Pfeiffer (Ed.), APA Handbook of Giftedness and Talent (pp. 185-199). Washington, DC: American Psychological Association. doi:10.1037/0000038-012.

Ribeiro, W. J., Nakano, T. C., \& Primi, R. (2014). Validade da estrutura fatorial de uma Bateria de Avaliação e Altas Habilidades. Psico, 45(1), 100-109. doi:10.15448/1980-8623.2014.1.13636

Rodrigo-Ruiz, D., Cejudo, J., \& Pérez-González, J. C. (2019). Compendio y análisis de medidas de evaluación de la inteligencia emocional capacidad. Revista Iberoamericana de Diagnóstico y Evaluación - e Avaliação Psicológica, 2(51), 99-115. doi:10.21865/RIDEP51.2.08.

Souza, A. C., Alexandre, N. M. C., \& Guirardello, E. B. (2017). Propriedades psicométricas na avaliação de instrumentos: Avaliação da confiabilidade e da validade. Epidemiologia e serviços de saúde, 26(3), 649-659. doi:10.5123/S1679-49742017000300022.

Sternberg, R. J. (2018). Theories of intelligence. In S. I. Pfeiffer (Ed.), APA Handbook of Giftedness and Talent (pp. 145-161). Washington, DC: American Psychological Association. Washington, DC: American Psychological Association. doi:10.1037/0000038-010.

Stoeger, H., Balestrini, D. P., \& Ziegler, A. (2018). International perspectives and trends in research on giftedness and talent development. In S. I. Pfeiffer (Ed.), $A P A$ Handbook of Giftedness and Talent (pp. 2537). Washington, DC: American Psychological Association. doi:10.1037/0000038-002.

Subotnik, R. F., Olszewski-Kubilius, P., \& Worrell, F. C. (2018). High performance: The central psychological mechanism for talent development. In S. I. Pfeiffer (Ed.), APA Handbook of Giftedness and Talent (pp. 0720). Washington, DC: American Psychological Association. doi:10.1037/0000120-002.

Veiga, $\quad$ E.C. (2014). Altas habilidades/superdotação e a psicopedagogia modular: Avaliando potencialidades. Revista Educação Especial, 27(50), 641-648. doi:10.5902/1984686X14283.

Vieira, N. J. W. (2014). Identificação pela provisão. Revista Educação Especial, 27(50), 699-712. doi:10.5902/1984686X14324.

Virgolim, A. M. R. (2013). A identificação de alunos para programas especializados na área das altas habilidades/superdotação: Problemas e desafios. Revista Brasileira de Altas Habilidades/Superdotação, 1(1). Retirado de: http://www.revistaconbrasd.org/wp/?p=94.

Zaia, P. \& Nakano, T. C. (2014). Escala de Identificação de Características associadas às Altas Habilidades/Superdotação (EICAH/S). Campinas, SP.

Zaia, P. Nakano, T. C., \& Peixoto, E. M. (2018). Scale for Identification of Characteristics of Giftedness: Internal structure analysis. Estudos de Psicologia (Campinas), 35(1), 3951. doi:10.1590/1982-02752018000100005.

Ziegler, A., Stoeger, H., \& Vialle, W. (2012). Giftedness and gifted education: The need for a paradigm change. Gifted Child Quarterly, 56(4), 194-197. doi:10.1177/0016986212456070.

Wellisch, M.. \& Brown, J. (2012). An integrated identification and intervention model for intellectually gifted children. Journal of Advanced Academics, 23(2), 145-167. doi:10.1177/1932202X12438877. 